\title{
High sensitivity troponin testing: is it what we really wanted?
}

\author{
Deborah B. Diercks \\ Department of Emergency Medicine, University of Texas Southwestern Medical Center, Dallas, TX 75390, USA.
}

Correspondence to: Prof. Deborah B. Diercks, Department of Emergency Medicine, University of Texas Southwestern Medical Center, 5323 Harry Hine Blvd, Dallas, TX 75390, USA. E-mail: Deborah.Diercks@UTSouthwestern.edu

How to cite this article: Diercks DB. High sensitivity troponin testing: is it what we really wanted? Vessel Plus 2021;5:52. https://dx.doi.org/10.20517/2574-1209.2021.113

Received: 19 Aug 2021 Accepted: 1 Sep 2021 Published: 5 Sep 2021

Academic Editor: Alexander D. Verin Copy Editor: Xi-Jun Chen Production Editor: Xi-Jun Chen

Diagnostic tests should be created to provide clinicians with the information can in decision-making. As a clinician, one must differentiate between a good diagnostic test and a good biomarker assay and provides adequate information to decide a diagnosis in the appropriate clinical setting. For example, an elevated lipase in a patient with mid-abdominal pain aids in the diagnosis of pancreatitis. The first-generation cardiac troponins, when introduced into the clinical area, were a good diagnostic test. Elevated cardiac troponin I and troponin T were highly suggestive of coronary artery disease. As the sensitivity of the assays for cardiac troponin has improved, the interpretation as a diagnostic test has become more complex. This increased sensitivity we see in current generations of hs-troponins has allowed the identification of myocardial injury. However, this myocardial injury is not limited to damage caused by the acute coronary syndrome. Because of the broad use of cardiac troponin testing in the acute care setting, hs-troponin must be carefully interpreted, and all reasons for elevation should be considered.

It is important to recognize that the value of hs-troponin as a biomarker extends beyond its function as a diagnostic test. Prior studies have shown that it has value as a prognostic indicator in patients with symptoms suggestive of an acute coronary syndrome ${ }^{[1,2]}$. These studies show that elevations above the 99th percentile are associated with an increased risk of major adverse cardiac events when compared with patients with levels below this threshold. The risk of major adverse cardiac events increases as hs-troponin levels increase. In diverse patient populations, the elevation of contemporary troponin has been associated with increased morbidity. In this edition, Conway et al. ${ }^{[3]}$ showed the value of hs-troponin as a prognostic 
indicator in a diverse emergency department patient population, excluding patients admitted for the acute coronary syndrome. This group reports that even after adjusting for other predictors of mortality, there is a linear relationship between hs-troponin and 30-day mortality. This analysis adds to the current body of evidence in that it excluded patients admitted for an acute coronary syndrome and continues to highlight the value of hs-troponin as a prognostic marker ${ }^{[1]}$.

The prognostic value of hs-troponin has also been considered in the evaluation of post-operative patients. In their review of the use of hs-troponin in the perioperative management of non-cardiac patients, Pal et al. ${ }^{[4]}$ discuss the known increased risk of mortality in patients with myocardial injury after non-cardiac surgery (MINS). In addition, they discuss recommendations for treatment in patients with MINS. Since it has been reported that up to $1 / 3$ of all non-cardiac surgery patients may have elevated hs-troponin, comparing pre-operative values to post-operative values may be important in the diagnosis of MINS ${ }^{[5]}$. As the incorporation of hs-troponin into clinical practice continues to grow, guidelines will need to be updated to include recommendations for timing and use of hs-troponin to identify MINS. As understanding of postoperative elevation increases, protocols will need to be derived and implemented that incorporate timing of measurements and treatment recommendations.

Essential to the implementation of protocols using hs-troponin is the need to ensure that clinicians understand how to interpret elevations in hs-troponin. Compared to prior generations of troponin, of hstroponin has been shown to increase the diagnosis of Type II myocardial infarction and has resulted in guidelines defining elevations above the 99th percentile as acute and chronic myocardial injury ${ }^{[6]}$. Implementation requires multi-disciplinary collaboration, including pathologists, hospitalists, cardiologists, nursing, and emergency physicians. Winchester ${ }^{[7]}$ discuss key points in their implementation process, highlighting the need for significant planning and education of providers. One key point was providing information on interpreting the abnormal values using absolute and delta values ${ }^{[7]}$. Another aspect that must be considered as the utilization of hs-troponin becomes standard across institutions is accounting for differences in hs-troponin assays. As patients from different institutions utilize different hs-troponin assays, clinicians need to understand the correlation between measurements. McDonald et al. ${ }^{[8]}$ reported the agreement between hs-troponin I and T assays and noted that $20 \%$ of all specimens tested had results abnormal by one assay and normal by the other. Clinicians making disposition decisions must understand that potential discordance exists if provided results from 2 different assays.

The introduction of hs-troponin into clinical practice provides the opportunity to enhance our ability to diagnose myocardial infarction and injury. However, as a diagnostic test, it introduces complexity that increases the cognitive burden on clinicians. In addition, the increased sensitivity of hs-troponin requires increased responsibility by the ordering clinician with consideration of alternative causes of elevations, understand their protocols for interpretation, and understand prognostic information. For clinicians that are uncomfortable with diagnostic tests that may require comparison to prior values and consideration of numerous causes of elevation, hs-troponin is not the test they wanted.

\section{DECLARATIONS}

\section{Authors' contributions}

The author contributed solely to the article.

\section{Availability of data and materials}

Not applicable. 


\section{Financial support and sponsorship}

Institutional Research Grants: Abbott, Roche, Siemens, Quidel, Ortho Diagnostics, Stage.

\section{Conflicts of interest}

The author declared that there are no conflicts of interest.

\section{Ethical approval and consent to participate}

Not applicable.

\section{Consent for publication}

Not applicable.

\section{Copyright}

(c) The Author(s) 2021.

\section{REFERENCES}

1. Chapman AR, Lee KK, McAllister DA, et al. Association of high-sensitivity cardiac troponin I concentration with cardiac outcomes in patients with suspected acute coronary syndrome. JAMA 2017;318:1913-24. DOI PubMed PMC

2. Nestelberger T, Boeddinghaus J, Wussler D, et al; APACE Investigators. Predicting major adverse events in patients with acute myocardial infarction. J Am Coll Cardiol 2019;74:842-54. DOI PubMed

3. Conway R, Byrne D, Cournane S, O’Riordan D, Coveney S, Silke B. Troponin status predicts 30-day in-hospital mortality. Vessel Plus 2020;4:25. DOI

4. Pal N, Worrall E, Lim A. Controversies in perioperative troponin elevation in non-cardiac surgery. Vessel Plus 2021;5:20. DOI

5. Devereaux PJ, Biccard BM, Sigamani A, et al; Writing Committee for the VISION Study Investigators. Association of postoperative high-sensitivity troponin levels with myocardial injury and 30-day mortality among patients undergoing noncardiac surgery. JAMA 2017;317:1642-51. DOI PubMed

6. Thygesen K, Alpert JS, Jaffe AS, et al; Executive Group on behalf of the Joint European Society of Cardiology (ESC)/American College of Cardiology (ACC)/American Heart Association (AHA)/World Heart Federation (WHF) Task Force for the Universal Definition of Myocardial Infarction. Fourth universal definition of myocardial infarction (2018). Circulation 2018;138:e618-51. DOI PubMed

7. Winchester DE. Implementation of high sensitivity troponin in a veterans health system: lessons learned. Vessel Plus 2021;5:1. DOI

8. McDonald S, Furmaga J, Vigen R, et al. Assessment of agreement of two high sensitivity troponin assays during an institutional transition. Vessel Plus 2021;5:38. DOI 